\title{
Research on chloride transport of prestressed box girders under coupling action of salt fog and fatigue loading
}

\author{
Guofang.Chen ${ }^{1, a}$, Yingzi. Zhang ${ }^{\star 1, b}$ and Chengxin.Wang ${ }^{1, ~ c}$, Lizhe.Jia, \\ Yiming.Duan
}

\author{
${ }^{1}$ Department of Civil Engineering, Harbin Institute of Technology; 264209; China \\ aemail: gfch@hit.edu.cn, bemail:zhyz@hit.edu.cn, cemail:wangchengxin@hit.edu.cn
}

Keywords: fatigue loading; salt fog area; prestressed box girders; coupling action.

Abstract: In order to research on the transport of chloride ions in prestressed box girders under coupling action of salt fog and fatigue loading, the volumetric strain and porosity as the intermediate variables, coupled processes was established based on the fatigue damage criterion. And compared with the related test data, the chloride ion distribution characteristics in the prestressed concrete of box girders under different prestress level and coupling times was investigated. The results showed that the coupling action significantly increases the chloride ion distribution in the surface of the prestressed rebar; Prestressed levels have little impact on the chloride ion permeability on the inside of the box girder, appropriately raise prestressed level can improve the permeability resistance of box girder; Coupling calculation results is less than a single coupling, and with the increase of penetration depth, multiple coupled with the computational result of single coupling gap is reduced, and which results is linear decreasing; Coupling times get basic guarantee for 10 times can calculation accuracy.

\section{Introduction}

Due to the rapid development of economy, the construction of the railway bridge at china coastal areas entered a new period. It is well known that the performance of bridge is critically affected by the loads (such as vehicles, people, waves, earthquakes, etc.), and the various erosion ions (such as chloride ions, sulfate ions, magnesium ions, etc.) under marine environment. In the past, many bridge under salt spray area appear have been significantly damaged. For example, the prefabricated beams were corrosion seriously under the splash area of the second Mateo-Hayward Cross-sea Bridge in San Francisco Bay San built in the 1960s, and had to spend huge huge amounts of money to repair after only 20 years' service [1]. There have many sea bridge damaged seriously and had to repair after several years' service, such as Norway's Gimsystraumen Bridge, the United States Alsea multi-arch bridge, the bridge on the Tarnell River in India, etc., which resulting in significant economic losses [1-3]. Coupling action of the chloride ions erosion in salt fog and fatigue loading are the main reasons resulting in the bridge damage, which lead to the corrosion of reinforcement and the loss of protective layer, and greatly reducing the durability of the bridge. At present, there are only a limited number of studies on the coupling action of fatigue load and chloride ion erosion in salty fog area, while most of the scholars considered the fatigue stress level as the main affect factor, and some experiment research was carried out to detect the coupling action of fatigue load and chloride ion erosion in the saturated zone and the splash zone [4-9]. The results indicate that compared with the static load, concrete beam under the fatigue loads has more serious corrosion and deterioration. Due to the limitation of experimental conditions, the prestress level was not considered. However, most of the box girders of the railway bridge under salty fog area are the prestressed box girder. Therefore, considering the coupling action of fatigue loading, prestressing force and salt spray erosion was very important, it has great significance to study the durability of prestressed box girder, extend the service life of bridge structure, improve the safety and durability of the structure under harsh environment, save resources, maintain the smooth operation of the railway network, avoid accidents and other aspects.

Based on the damage theory of strain, this paper takes the volume strain and porosity as intermediate variables to establish the coupling simulation method of fatigue loading and chloride ion 
erosion, and then the numerical simulation method is validated by comparing the experimental results. At last, a fine model of prestressed concrete railway bridge box girder is established to detect the distribution of the chloride ion mass fraction under various prestress levels and coupling action of fatigue-erosion.

\section{Numerical simulation of chloride ion transfer under the coupling action of fatigue and salt spray}

\section{Basic principle of the simulation method.}

Concrete strain will be generated when the load applied. With the accumulation of strain will cause the volume expansion and volume compression, which lead to the change of micro cracks and porosity in concrete. The transport principles of chloride ions in concrete will be changed with the microcracks extend to macroscopic cracks. Considering the possible effect of the damage on the chloride ion permeation, the coupling variable between the stress field and the chloride ion diffusion field is connected with the volume strain and the porosity under the loading, which can be studied the coupling action between the load and the chloride ion erosion. According to the deduction of material mechanics in [4], Eq. (1) can be obtained:

$$
\varphi=\frac{\varepsilon_{v}+\varphi_{0}}{1+\varepsilon_{v}}
$$

Where $\varphi_{0}$ is the porosity of concrete before load; $\varepsilon_{v}$ is the volume strain, which reflects the nature of concrete materials, there has no connection with the load type, it can be applied to all the load.

According to the literature [4], the integrated chloride diffusion coefficient can be written as Eq.(2)

$$
D^{*}=\frac{\lambda D_{r f}}{1+\frac{1}{\omega} \cdot \frac{\partial \rho_{b}}{\partial \rho_{f}}} \cdot\left(\frac{t_{28}}{t}\right)^{\mathrm{m}} \cdot \exp \left(\frac{U}{R} \cdot\left(\frac{1}{T_{\mathrm{rf}}}-\frac{1}{T}\right)\right) \cdot\left[1+\frac{(1+s)^{4}}{\left(1+s_{0}\right)^{4}}\right]^{-1}
$$

Where $m$ is the time dependence index of diffusion coefficient; $t$ is the service time, $t_{28}=28 d$; $D_{\text {ref }}$ is the fitting results of the chloride ion diffusion coefficient and porosity in the mortar matrix based on the experiment result be drawn by Garboczi et al. [10].

$$
D_{\text {ref }}=D_{0} \cdot\left(0.001+0.07 \varphi+1.8 H \cdot\left(\varphi-\varphi_{\text {th }}\right)^{3}\right)
$$

Where $D_{0}$ is the chloride ion diffusion coefficient in aqueous solution, $D_{0}=2.032 \times 10^{-9} \mathrm{~m}^{2} / \mathrm{s} ; \varphi$ is the porosity of mortar matrix; $H$ is Heaviside function, when $\varphi>\varphi_{t h}, H=1$, otherwise $\mathrm{H}=0 ; \varphi_{t h}$ is the threshold of porosity, $\varphi_{t h}=0.18$.

According to the relationship between comprehensive diffusion coefficient $D^{*}$ and porosity $\varphi$ of concrete material show in Eq. (2), it can establish the relationship between volume strain and porosity, and then it can be obtained the coupling action of load and chloride erosion based on the relationship between porosity and chloride ion diffusion coefficient, the solution flow chart shown in Figure 1.

\section{Basic principle of simulated the fatigue-chloride ion erosion coupling action.}

With the microcracks expand and the damage increases, concrete internal will form macroscopic cracks under the repeated action of fatigue load. The cumulative residual strain can be used to characterization the cumulative damage of structure. Based on the available experimental data [11], the strain calculation Eq.(4) of concrete under fatigue load can be obtained by Guojun Liu with regression analysis.

$$
\varepsilon_{r}(N)=\varepsilon_{r}(l)+\frac{0.00105 \varepsilon_{\max }^{1.98}\left(1-\frac{\varepsilon_{\text {min }}}{\varepsilon_{\text {max }}}\right)^{5.27}}{\varepsilon_{\text {unsab }} 1.41} N^{0.395}
$$

Where $\varepsilon_{r}(N)$ the residual strain of sample under $N$ is times fatigue load; $\varepsilon_{\min } . \varepsilon_{\max }$ is the lower limit and the upper limit transient strain of sample under fatigue load; $\varepsilon_{r}(l)$ is the residual strain after the time stress cycle, $\varepsilon_{r}(l)=0.25\left(\varepsilon_{\max } / \varepsilon_{\text {unstab }}\right)^{2} ; \varepsilon_{\text {unstab }}$ is the strain of concrete under the unstable state, and its value approximately equal to $\varepsilon_{0}$ and generally is 0.002 . 
The damage degree of concrete under the fatigue load at a certainly stress level can be expressed by the Equation $D_{\mathrm{F}}=\varepsilon_{r}(N) / \varepsilon_{\text {unstab }}$, where each variables are the same to Eq. (4). The total strain of structure is $\varepsilon_{\max }(N)=\varepsilon_{\max }+\varepsilon_{r}(N)$ after $N$ times fatigue load, therefore, the residual strain can be obtained by the damage degree, and to calculate the total strain under the certainly stress level cycle and the number of cycles. For the variable-amplitude fatigue, the total strain by a direct linear superposition (shown in Eq.(5) based on the "unique" assumption of deformation, and the specific damage degree and the total residual strain can be got under the variable-amplitude fatigue.

$$
D_{F}=\sum_{i} D_{i}=D_{1}+D_{2}+\ldots+D_{n}=\frac{\varepsilon_{r}\left(N_{1}\right)}{\varepsilon_{\text {uustab }}}+\frac{\varepsilon_{r}\left(N_{2}\right)}{\varepsilon_{\text {unsab }}}+\ldots+\frac{\varepsilon_{r}\left(N_{n}\right)}{\varepsilon_{\text {unsab }}} .
$$

From the Eq.(5), it can be observed that the residual strain can be deduced by the damage of the structure, and then the total volume strain and the chloride ion diffusion coefficient can be obtained, finally, the chloride ions distribution in concrete structure can be got under the coupling action of fatigue load and chloride ion.

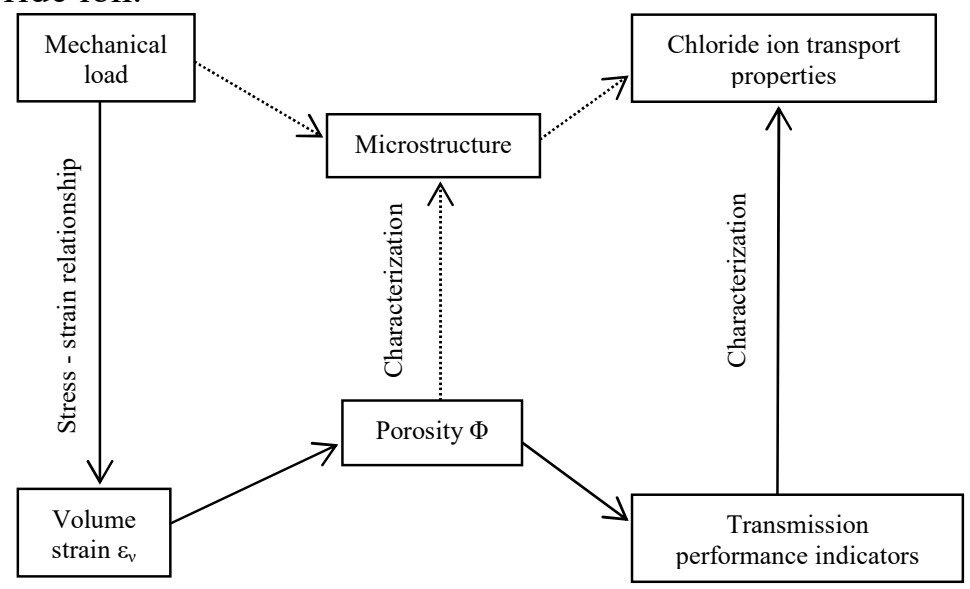

Fig.1 Chloride ion transport method under the coupling action of load and chloride erosion

\section{Verification of simulation method for coupling action of fatigue load-chloride ion erosion.}

Based on the test by Peihua Sun [5] to verify the effectiveness of the simulation method. The sample size is $100 \mathrm{~mm} \times 100 \mathrm{~mm} \times 200 \mathrm{~mm}$. Compressive strength is $33.9 \mathrm{MPa}$ after 28 days standard curing. Four various load levels of concrete (respectively $0,0.4,0.6,0.8$ ) be measured. In this paper, the finite element software ANSYS was conducted to establish and calculate the structural model and thermal model, and the fatigue analysis module is used to solve the damage degree.

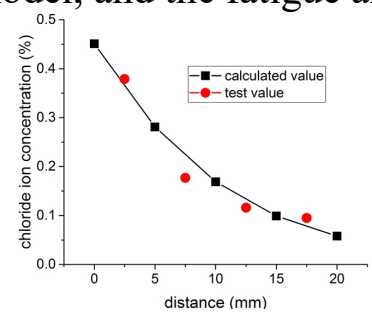

a) No load

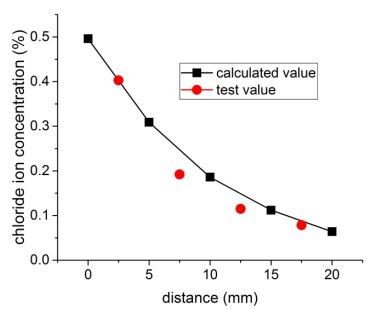

b)Load level $=0.4$

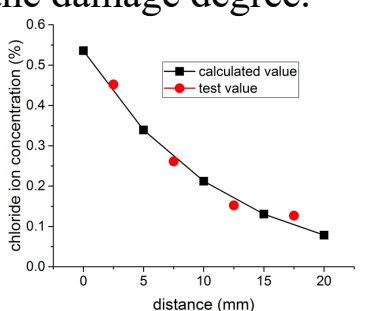

c)Load level $=0.6$

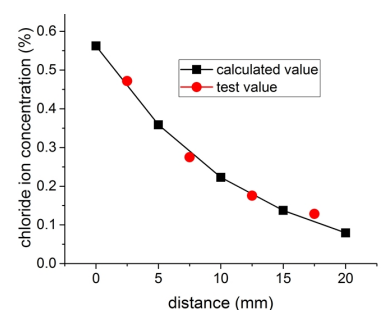

d)Load level $=0.8$

Fig. 2 The results of numerical simulation and experiment after 5 cycles of cyclic loading

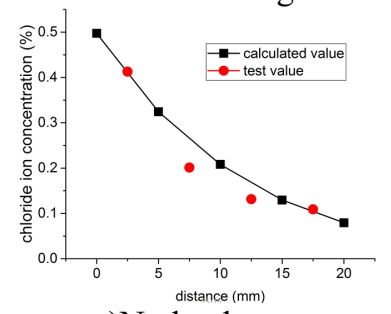

a)No load

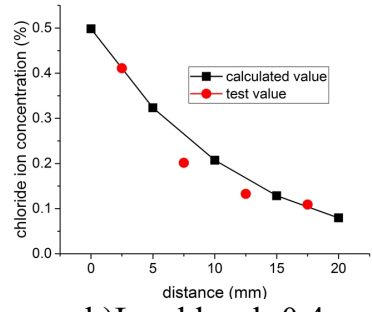

b)Load level $=0.4$

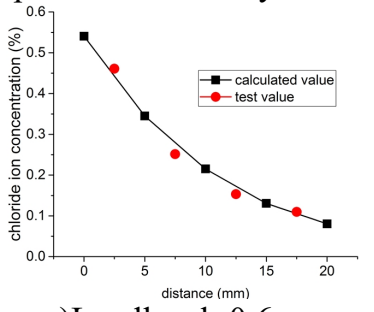

c)Loadlevel $=0.6$

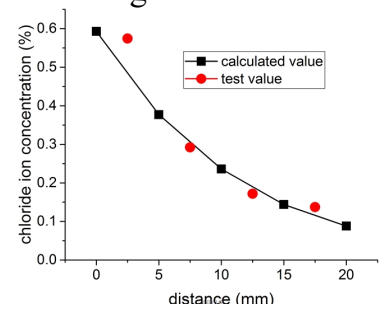

d)Load level $=0.8$

Fig. 3 The results of numerical simulation and experiment after 25 cycles of cyclic loading

Fig. 2 and Fig. 3 presents the results of numerical simulation and experimental value of chloride ions permeability in concrete under various load level and cycle times of fatigue load. It can be concluded that the calculated result is coincide with the experimental results, which verifies the effectiveness of the simulation method. 


\section{Numerical simulation of chloride Ion transport in prestressed box girder with coupling}

\section{Establishment of numerical model of reinforced concrete box girder.}

According to the literature [12-13], the box girder is a single box, single chamber, variable cross section and contour structure, the structure dimensions are shown in Table 1. Arrangement of prestressed steel bars are shown in Fig4.

Table 1 structure size of beam

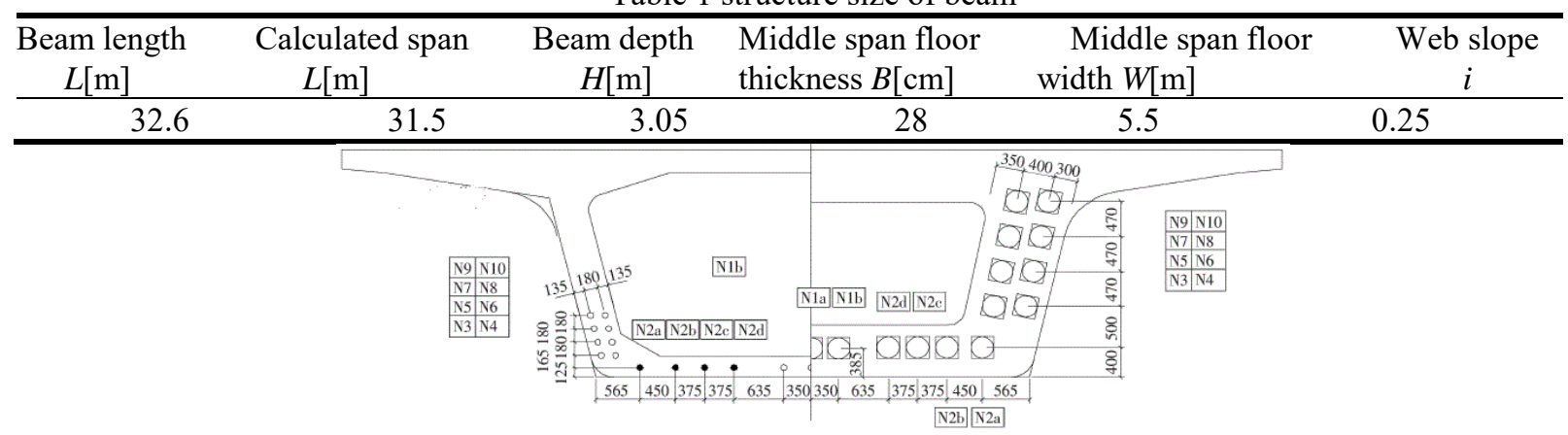

Fig.4 Arrangement of transverse section of prestressed steel bar

The prestressing tendons at the N1a are $8 \varphi 15.2$, the other locations are $9 \varphi 15.2$. The design tensile strength of the steel strand is $1860 \mathrm{Mpa}$. The stretching prestressed at N1a、N2a、N2c is $1563.4 \mathrm{kN}$; at $\mathrm{N} 2 \mathrm{~b}, \mathrm{~N} 2 \mathrm{~d}$ is $1757.7 \mathrm{kN}$, at N4, N6, N8, N10 is $1786.84 \mathrm{kN}$. Based on the actual results of the field in the literature[12], the materials basic attributes and simplified results used are shown in Table 2 .

During the structural calculation stage of ANSYS, the prestressed steel bar is modeled by Link8 unit; the constitutive relation is the bilinear follow-up model; the brittle (elastic and plastic slope) is used to represent the stress-strain curve of steel bar. The steel elastic modulus is $210 \mathrm{Gpa}$; the Poisson's ratio is 0.3 ; the strand yield strength is $1580 \mathrm{Mpa}$, and the shear modulus of second line of the bilinear is $2.1 \mathrm{Gpa}$.

Table 2 Material basic attributes

\begin{tabular}{ccccc}
\hline Material type & $\begin{array}{c}\text { Elastic modulus } \\
E[\mathrm{Mpa}]\end{array}$ & $\begin{array}{c}\text { Poisson's } \\
\text { ratio }\end{array}$ & $\begin{array}{c}\text { Axial tension strength } \\
f_{\mathrm{t}}[\mathrm{Mpa}]\end{array}$ & $\begin{array}{c}\text { Axial compression } \\
\text { Strength } f_{\mathrm{c}}[\mathrm{Mpa}]\end{array}$ \\
\hline $\begin{array}{c}\text { C50 concrete } \\
\text { Ordinary }\end{array}$ & $4.64 \mathrm{e} 4$ & 0.2 & 7.2 & 72 \\
$\begin{array}{c}\text { reinforcement } \\
\text { Concrete with steel }\end{array}$ & $2.1 \mathrm{e} 5$ & 0.3 & 200 & 200 \\
\hline
\end{tabular}

Solid65 units are used to simulate the concrete. Constitutive relationship is the multi-linear isotropic strengthening model. The stress-strain curve of concrete under uniaxial compression deduced by Rush is applied in this paper, the expression is shown in Eq.(6):

$$
\left\{\begin{array}{cc}
\sigma=f_{c} \cdot\left[2 \varepsilon / \varepsilon_{0}-\left(\varepsilon / \varepsilon_{0}\right)^{2}\right] & \varepsilon \leq \varepsilon_{0} \\
\sigma=f_{c} & \varepsilon_{0} \leq \varepsilon \leq \varepsilon_{u}
\end{array} .\right.
$$

Considering the calculation efficiency, the two-dimensional element PLANE55 are taken into during the calculation of thermal module. To evaluate the fatigue effects, the $S-N$ curve of the concrete is defined in the fatigue analysis. The Eq. (7) proposed by Aas Jakobsen [14] are accepted.

$$
S_{\max }=1-\beta(1-R) \lg N
$$

Where $S_{\max }$ is the fatigue strength of concrete, generally, represented by the static strength ratio, $S_{\max }=\sigma_{\max } / f_{\mathrm{cm}} ; \beta$ is the material constant, according to the test results of 475 pieces by Tepfers and Kutti et al [15], it can be seen that $\beta=0.0685$, this value applies to the ordinary concrete and lightweight aggregate concrete.

\section{Define the input parameters of thermic calculation .}

When the permeation time less than 20 years, the chloride ion concentration on the surface of the concrete in the salty fog area is a variable with the time changed. Therefore, the chloride ion concentration on the surface should be considered while to study the distribution of chloride ion inside box girder. According to the result of literature [16], the model of chloride concentration on the 
surface of concrete under salty fog area as shown in Eq. (8). When the permeation time more than 20 years, the surface chloride concentration tends to be stable and the $C_{s}$ is $0.05429 \%$.

$$
C_{s}=\left\{\begin{array}{cc}
{[-1.062 \exp (-t / 4.873)+1.018] \times 0.051} & t \leq 20 \\
0.05429 & t>20
\end{array}\right.
$$

According to literature [16], the chloride ion diffusion coefficient of unsaturated concrete is $1.92 \times 10^{-8} \mathrm{~cm}^{2} / \mathrm{s}$ under coupling action of diffusion and convection.

Influence of prestress level on structure under fatigue - salty fog coupling.

Considering the fatigue-chloride ion erosion coupling, the original prestressing force of the box girder model is set to 1.0 times. The different prestressing levels of $0 、 0.4$ and 0.8 by changed in the model. The relationship between the chloride ion mass fraction and permeation time at the same distance from exposed surface of box girder is shown in Fig.5. The chloride ion mass fraction of each layer under various prestressing levels after 30 years exposure is listed in Table 3.

Table 3 The chloride ion mass fraction of each layer under various prestressing levels after 30 years exposure

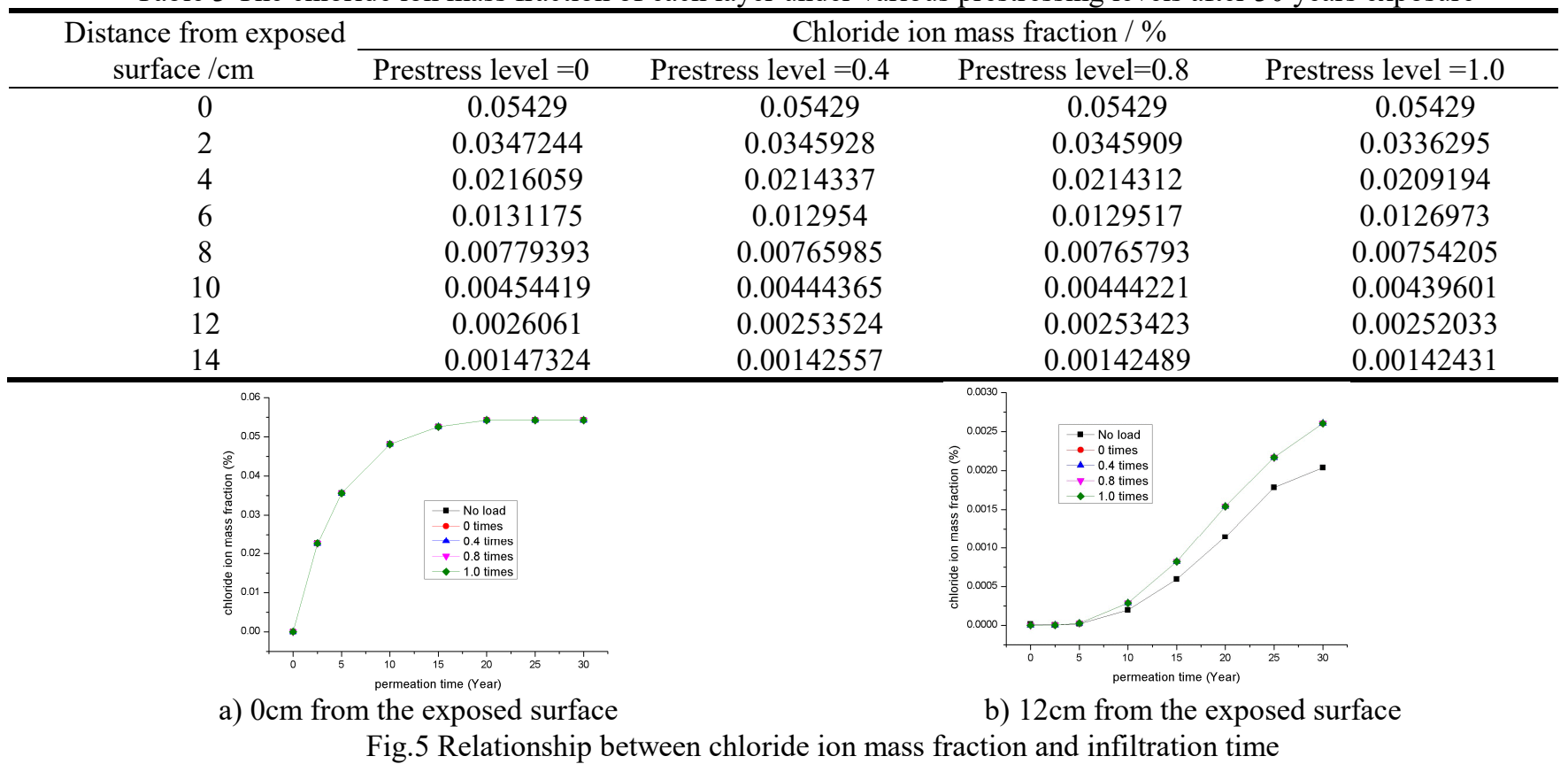

From the Fig.5, it is obvious that the calculation result with the coupling less than the result with other factors, and with the increase of distance from the exposure surface, the difference is larger. The result of the chloride ion concentration on the surface of prestressed tendons at a distance of $12 \mathrm{~cm}$ away from the exposed surface is seriously decreased without the fatigue load and the chloride ion erosion coupling. The difference of the two conditions can be up to $24.6 \%$. In addition, the influence of the different prestressing levels is negligible while considering the load. Therefore, the effect of the prestressing level can be ignored during the calculation.

As shown in Table 3, the lower prestress level, the slightly higher calculation result of chloride ion concentration. Therefore, there has a slight impact of the prestressing level on the calculation results by the above analysis. From the related research [13], it can be finding that the effect of various prestressing level on the deflection and other displacement results will be decreased distinctly of concrete box girder with the external load (such as the ZK live load, that is the static load) be applied.

\section{Numerical simulation of chloride transport in prestressed box Girder under multiple coupling}

\section{Method of primary principle of multiple coupling calculations.}

In the nature engineering, fatigue and erosion affect each other. ANSYS can just achieve the coupling simulation from the structure to the thermic in simulation. which is different from the nature coupling. But the coupling process is divided into several coupling of the aforementioned fatigue-chloride coupling, scilicet the process of fatigue-infiltration-fatigue-infiltration-fatigue-infiltration..., this 
promote the result of the calculation to approach to the nature coupling. The key procedure of multiple coupling of fatigue load - chloride infiltration is listed as follows: firstly, the calculation time is divided into $\mathrm{n}$ times. The cumulative fatigue damage coefficient $D_{i}$ of the structure can be calculated by ANSYS after the $i$-th time fatigue accumulated. And then, the cumulative residual strain of the structure is calculated according to the cumulative fatigue damage coefficient $D_{i}$, and the volume strain of structure can be gotten during the $i$-th time period. The revised chloride ion diffusion coefficient $D * i$ during the $i$-th time period can be obtained base on the the $i$-th time volume strain. At last, utilize the thermal analysis module of ANSYS to find out the distribution of chloride ions inside the box girder with the determined transfer coefficient $D_{i}$ and surface chloride ion concentration $C_{s i}$ at the $i$-th time period. Finally, the multiple coupling of fatigue process - chloride ion permeability can be completed with repeating the aforementioned process of coupling.

\section{Results of single and multiple coupling calculation.}

According to the aforementioned ideas, the volume strain, porosity and transfer coefficient of the structure are obtained, and the thermal analysis model o structure to analyze the of the box girder is established. To explore the regularity of chloride ion permeability of structure set the coupling at 5 and 10 times, the period of simulation at 30 years. The decreasing amplitude of simulation results with multiple coupling and single coupling are shown in Fig. 6.

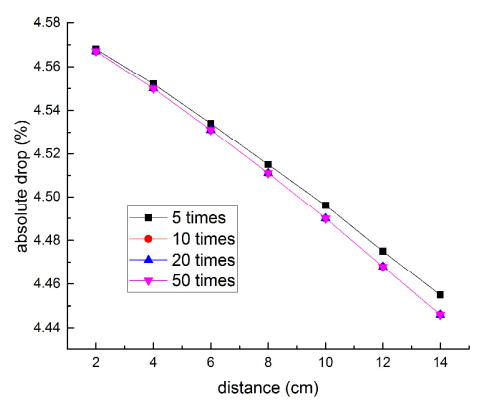

Fig.6 The results under multiple coupling and single coupling after 30 years permeated (absolute value)

From Fig.6, it can be dawn that the decreasing amplitude of results with 5 and 10 times coupling are $4.568 \%$ and $4.567 \%$, respectively. With the increase of infiltration depth, the difference between the results of multiple coupling and single coupling is reduced and basically linearly decreasing. And the results of multiple coupling are slightly smaller than that of single coupling. Increased coupling times lead to increased computational results. The results of coupling 10 and 20 times are basically no difference, while the number of coupling exceeds to 50 times, the calculation result will be further enhanced. However, the increasing amplitude has been small and can be ignored. Due to the calculation efficiency, the calculation accuracy can be effectively guaranteed when the coupling times be set to 10 .

\section{Conclusions}

1) The simulation results of coupling action between static load and chloride ion erosion base on the volume strain and porosity as the intermediate variable are coincide with the experimental results. Fatigue damage of structure is calculated by using the fatigue module in ANSYS, and then the total volume strain under fatigue load can be deduced. The method of calculating the total volume strain under the fatigue load is effectively available to simulate the coupling action of fatigue load and chloride ion erosion

2) After 30 years permeated, with the increase of diffusion depth and time, the distribution of chloride ions in box girder with coupling action is gradually larger than that without coupling action. The difference of simulation results between with and without coupling action can be up to $24.7 \%$ on the surface of prestressed tendons.

3) The chloride ion distribution with various prestressing level sustained a smaller change under the external loading. It can be noted that the higher prestressing level, the better impermeability of box girder. 
4) With the increase of coupling times of load and chloride ion erosion, the chloride ion concentration at each depth is lower than that of single coupling. With the increase of penetration depth, the difference of simulation results between multiple coupling and single coupling gradually reduced, and basically linearly decreasing. After 30 years permeated, there is a certain difference of the calculation results with 5,10,20,50 times coupling. The calculation results gradually decreased with the coupling times added. While the coupling exceeds to 10 times, the reduction is minimal. In order to guarantee the calculation accuracy, the coupling times can be set to 10 .

\section{Acknowledgements}

This work was financially supported by the National Natural Science Foundation of PR.China (51208155, 51308166) and Weihai Science and technology Development Plan Project (2013DXGJ08, 2015DXGJMS011).

\section{References}

[1] Pasko T J.: Proceedings of ACI International Conference on High-Performance Concrete. Singapore, 1994, p.123-134.

[2] Fluge F.: submitted to Theory to Practice in a Marine Environment (1997).

[3] L.Zheng, Beaudoin J J. submitted to Concrete and Aggregates (2000) .

[4] P.H.Sun, in: Master Dissertation of Dalian University of Technology, Dalian, (2012).

[5] J.X.Wu, in: Master Dissertation of Harbin Institute of Technology, Harbin (2012).

[6] Nakhi A E, Xi Y and Willam K.: European Congress on Computational Methods in Applied Sciences and Engineering, Barcelona, 2008, p.1-8.

[7] Xi Y P, Ababneh A.: Proceedings of the International Conference ICACS, 2003, p.354-365.

[8] Saito M, Ishimori H.: submitted to Cement and Concrete Research (1995).

[9] Jaffer S J, Hansson C M.: submitted to Cem Concr Res (2009).

[10] Garboczi E J, Bentz D P. Submitted to Advanced Cement Based Materials (1997).

[11] G.J.Liu, in Doctoral Dissertation of Southwest Jiaotong University, Chengdu (2008).

[12] A.Gao, in: Master Dissertation of Changsha University of Science \& Technology, Changsh (2010).

[13] C.L.Dong, in: Master Dissertation of Central South University, Changsha (2010).

[14] Aas-Jakobsen K.: submitted to NTH-Trondheim, Bulletion (1970).

[15] Tepfers R,Kutti T.: submitted to ACI Journal (1979).

[16] T.X.Xu, in: Master Dissertation of Harbin Institute of Technology, Harbin (2014). 